\title{
Low phase noise microwave generation from a direct-modulation optoelectronic oscillator (DM-OEO)
}

\author{
Brian Sinquin, Marco Romanelli, Steve Bouhier, Mehdi Alouini, Marc Vallet \\ Univ. Rennes, CNRS, Institut FOTON UMR 6082, F-35000 Rennes, France
}

Optoelectronic oscillators (OEO) have become of paramount importance for the generation of high spectral purity, (optically-carried) microwave signals. The most widespread architecture uses a Mach-Zehnder Modulator as the generator of the RF modulation [1]. Direct modulation of the injection current of a semi-conductor laser, arguably the simplest possible OEO [2], has been comparatively much less explored [3].

In the present contribution, we demonstrate a direct-modulation OEO with performances comparable to the standard external-modulation architecture, while reducing the system size and complexity.

In a first experiment we use a single loop architecture with a $5 \mathrm{~km}$ fibre spool. The laser is a commercial telecom 1.5 $\mu \mathrm{m}$ DFB laser designed for direct modulation. Its emitted power is detected by a PIN photodiode after propagation in the fibre. The photocurrent is then amplified and filtered by a high quality factor bandpass filter centred at $10 \mathrm{GHz}$, before being fed back to the pump current. A part of the signal fed back to the laser is extracted using a RF power splitter.
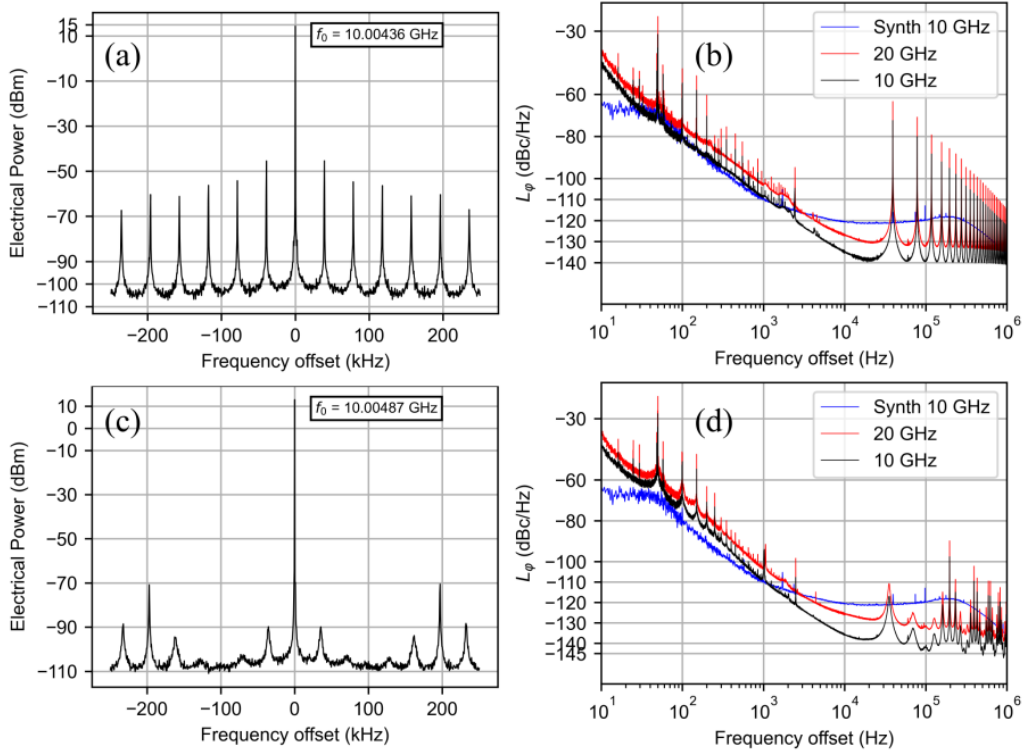

Fig. 1 (a,c) RF spectrum of the oscillator output for the single loop (a) and dual-loop (b) cases. (b,d) Phase noise for the single loop (b) and dual-loop (d) cases, for the $10 \mathrm{GHz}$ signal and for the $2^{\text {nd }}$ harmonic at $20 \mathrm{GHz}$. For comparison, the phase noise of a low-noise RF synthesizer (Rohde \& Schwarz SMF100A) is shown.

Stable RF oscillation at $10 \mathrm{GHz}$ is observed, with a rejection ratio of $60 \mathrm{~dB}$ and no mode hops (Fig. 1 (a)). We measure a phase noise of $-135 \mathrm{dBc} / \mathrm{Hz}$ at $10 \mathrm{kHz}$ from the carrier, and a noise floor of -140 dBc/Hz (Fig. 1 (b)). A dual-loop architecture, using two fibre spools of different lengths (5 and $6 \mathrm{~km})$ and two photodetectors in order to obtain a RF interferometer, improves some figures of the OEO: the rejection ratio of non-oscillating modes is $>80 \mathrm{~dB}$ (Fig. 1(c)), and the suppression of the phase noise of the closest-to-the-carrier spur is $>40 \mathrm{~dB}$ (Fig. 1 (d)) compared to the single-loop case. Thanks to the nonlinearity of the detection and amplification, a $20 \mathrm{GHz}$ signal of $-5 \mathrm{dBm}$ power is also available at the output of the oscillator, with a slight increase of the phase noise due to reduced power and frequency multiplication (Fig. 1(b, d)). To the best of our knowledge, these figures represent the state-of-the-art of DM-OEOs. The architecture based on direct modulation is thus a valuable alternative, promising in particular for monolithic integration in photonic chips [4].

\section{References}

[1] L. Maleki, "Optoelectronic oscillators for microwave and mm-wave generation”, IEEE 2017 18th International Radar Symposium (IRS), p. 1-5 (2017); Y. K. Chembo, D. Brunner, M. Jacquot, and L. Larger, "Optoelectronic oscillators with time-delayed feedback", Rev. Mod. Phys. 91, 035006 (2019).

[2] G. R. G. Chengui, P. Woafo, and Y. K. Chembo, "The simplest laser-based optoelectronic oscillator: An experimental and theoretical study", J. Light. Technol. 34, 873 (2016).

[3] H. K. Sung, X. Zhao, E. K. Lau, D. Parekh, C. J. Chang-Hasnain, and M. C. Wu, "Optoelectronic oscillators using direct-modulated semiconductor lasers under strong optical injection", IEEE J. Sel. Top. Quantum Electron. 15, 572 (2009).

[4] P. Primiani, H. Débrégéas, D. Lanteri, M. Alouini, and F. van Dijk, "Electro-Absorption modulator-based optoelectronic oscillator", IEEE, 2017 International Topical Meeting on Microwave Photonics (MWP), p. 1-3 (2017). 